\title{
Information Revelation and Efficiency in Auctions
}

\author{
Anna Mikusheva*and Konstantin Sonin ${ }^{\dagger}$
}

June 1, 2002

\begin{abstract}
We study the impact of information revelation on efficiency in auctions. In a constrained-efficient mechanism, i.e. a mechanism that is efficient subject to the incentivecompatibility constraint, any additional information available to bidders increases the expected efficiency of the mechanism. This result cannot be extended to a more general setup: in a second-price sealed bid auction, revelation of information might lead to efficiency losses.
\end{abstract}

\section{Introduction}

Optimal auctions are those that raise maximum revenue. Efficient auctions deliver the object to the bidder that values it the most. In a seminal paper, Milgrom and Weber (1982) showed that the best policy for the auctioneer is to fully reveal any relevant information about the good if the goal is to increase revenues. A natural question seems to be whether revelation of information is a good policy if the goal is to increase efficiency. ${ }^{1}$

The answer to this question might have profound policy implications. Suppose the government plans two sales, one on the East Coast and one on the West Coast, and suppose the sets of bidders are completely different. Still, it might well be that results (including the bidding history) of the auction on one coast bear information about the object being sold on

${ }^{*}$ Harvard University; mikouch@fas.harvard.edu.

${ }^{\dagger}$ New Economic School/CEFIR, Moscow; ksonin@nes.ru.

${ }^{1}$ Achieving higher efficiency rather than maximizing revenues was a stated goal in many recent auctions, such as the sale of telecommunication spectrum in the US and Britain. 
the other. Now, if the government runs the auctions sequentially, the bidders in the second one would have more information than if the auctions are simultaneous.

So, does more information lead to increased efficiency? A natural answer is probably 'yes.' The general logic might be as follows: the less uncertainty about the object bidders face, the less is the probability that the object goes to the wrong bidder. Auctions in environments with multidimensional signals are often inefficient because of the impossibility of efficient aggregation of multidimensional information in a one-dimensional bid (Dasgupta and Maskin, 2000; Jehiel and Moldovanu, 1998). ${ }^{2}$

There are two major obstacles to obtaining a general result. The first is the absence of a focal equilibrium in a general setting. This does not allow one to trace changes in efficiency due to additional information. ${ }^{3}$ The second difficulty is the absence of a symmetric equilibrium in many natural settings with multidimensional signals (Jackson, 1998; Pesendorfer and Swinkels, 1999). We consider constrained-efficient mechanisms, i.e. mechanisms that achieve the highest efficiency subject to the constraints of incentive-compatibility. We prove that disclosure of any additional information in a constrained-efficient mechanism leads to an increase in expected efficiency. However, this result cannot be extended to a general result that revealing information always leads to increasing social welfare for all types of auctions. We provide examples of situations, where revealing additional information worsens the equilibrium outcome, i.e. leads to efficiency losses or even to disappearance of a focal equilibrium. A possible explanation might be as follows: whereas the inefficiency caused by multidimensionality reduces with disclosure of information, the inefficiency caused by interdependence between signals agents possess can increase.

This rest of the paper is organized as follows. In Section 2, it is proved that in a constrained-efficient mechanism, revelation of information does increase efficiency. Section 3 contains some examples that demonstrate that this result cannot be extended to cover

\footnotetext{
${ }^{2}$ For example, suppose that both private and common component matter in bidders' valuations, and bidders know for sure their private components and observe privately some noisy signals concerning the common component. Then it is impossible to infer from a high bid whether the bidder has a high private component or is very optimistic about the common one. If only the private component matters for efficiency, then it is not always efficient to award the object to the bidder with the highest bid.

${ }^{3}$ Goeree and Offerman (1999) claim to obtain a result that disclosure of some additional information leads to an increase in efficiency. However, the authors' interpretation of additional information as an independent random summand in common value seems to provide limited intuition.
} 
second-price sealed-bid auctions. Section 4 concludes.

\section{The Theory}

To study effects of infromation disclosure on efficiency, we start with a general model. There are $N$ risk-neutral agents and one object for sale. Each agent receives a private multidimensional signal $s_{i} \in S_{i} \subset \mathbf{R}^{m}$, and all signals are independent. The value of the object for agent $i$ is $v_{i}=v_{i}\left(s_{1}, \ldots, s_{N}\right)$. We assume that buyer $i$ 's signal is separable between his own signal and the signals of others, i.e. there exist functions $\tau: \mathbf{R}^{m} \rightarrow \mathbf{R}$ and $\psi: \mathbf{R}^{m(N-1)+1} \rightarrow \mathbf{R}$ such that $v_{i}\left(s_{i}, s_{-i}\right)=\psi_{i}\left(\tau_{i}\left(s_{i}\right), s_{-i}\right)$ holds for all $\left(s_{i}, s_{-i}\right)$. Let $\Omega$ denote other information available to all bidders. For each vector $\left(t_{1}, \ldots, t_{N}\right) \in \mathbf{R}^{m}$, define

$$
w_{i}\left(t_{1}, \ldots, t_{N}\right)=E_{s_{-i}}\left[\psi_{i}\left(t_{i}, s_{-i}\right) \mid \tau_{-i}\left(s_{-i}\right)=t_{-i}, \Omega\right]
$$

A constrained-efficient mechanism delivers the object to the agent with the highest $w_{i}\left(t_{1}, \ldots, t_{N}\right)$ (Dasgupta and Maskin, 2000). We measure efficiency of a mechanism as the expected value of the object to the winner. For a constrained-efficient mechanism, this gives

$$
W(\Omega)=\sum_{i=1}^{N} E v_{i}(s) I\left\{w_{i}\left(\tau_{i}\left(s_{i}\right), \tau_{-i}\left(s_{-i}\right)\right)=\max _{j} w_{j}\left(\tau_{j}\left(s_{j}\right), \tau_{-j}\left(s_{-j}\right)\right)\right\},
$$

where $I\{\cdot\}$ is an indicator function.

Theorem 1 In a constrained-efficient mechanism, revealing publicly any additional information (weakly) increases efficiency.

Proof. We have

$$
\begin{aligned}
W(\Omega) & =E \sum_{i} E_{s_{-i}}\left[v_{i}(s) I\left\{w_{i}\left(\tau_{i}\left(s_{i}\right), \tau_{-i}\left(s_{-i}\right)\right)=\max _{j} w_{j}\left(\tau_{j}\left(s_{j}\right), \tau_{-j}\left(s_{-j}\right)\right)\right\} \mid \tau_{-j}\left(s_{-j}\right)=t_{-j}, \Omega\right] \\
& =E \sum_{i} E_{t_{-i}} w_{i}\left(t_{i}, t_{-i}\right) I\left\{w_{i}\left(t_{i}, t_{-i}\right)=\max _{j} w_{j}\left(t_{j}, t_{-j}\right)\right\} \\
& =E \max _{j} w_{j}\left(t_{j}, t_{-j}\right)=E \max _{j} E_{s_{-j}}\left[v_{j}\left(t_{j}, s_{-j}\right) \mid \tau_{-j}\left(s_{-j}\right)=t_{-j}, \Omega\right] .
\end{aligned}
$$

Now suppose that $\Omega^{\prime} \subset \Omega$, i.e. $\Omega$ provides more information about the object than $\Omega^{\prime}$. Then

$$
\begin{aligned}
W\left(\Omega^{\prime}\right) & =E \max _{j} E_{s_{-j}}\left[v_{j}\left(t_{j}, s_{-j}\right) \mid \tau_{-j}\left(s_{-j}\right)=t_{-j}, \Omega^{\prime}\right] \\
& =E \max _{j} E\left[E_{s_{-j}}\left[v_{j}\left(t_{j}, s_{-j}\right) \mid \tau_{-j}\left(s_{-j}\right)=t_{-j}, \Omega\right] \mid \tau_{-j}\left(s_{-j}\right)=t_{-j}, \Omega^{\prime}\right]
\end{aligned}
$$




$$
\begin{aligned}
& \leq E E\left[\max _{j} E_{s_{-j}}\left[v_{j}\left(t_{j}, s_{-j}\right) \mid \tau_{-j}\left(s_{-j}\right)=t_{-j}, \Omega\right] \mid \tau_{-j}\left(s_{-j}\right)=t_{-j}, \Omega^{\prime}\right] \\
& =E \max _{j} E_{s_{-j}}\left[v_{j}\left(t_{j}, s_{-j}\right) \mid \tau_{-j}\left(s_{-j}\right)=t_{-j}, \Omega\right]=W(\Omega),
\end{aligned}
$$

where the inequality follows from the fact that the expectation of maximum of random variables is at least as large as the maximum of their expectations.

Dasgupta and Maskin (2000) formulate sufficient conditions for the existence of a constrainedefficient mechanism. Example 1, based on Example 5 from Dasgupta and Maskin (2000), demonstrates how such a mechanism works in a simplest possible case, and shows that revelation of information strictly increases efficiency.

Example 1. Each of two agents privately receives a two-dimensional signal $\left(q_{i}, c_{i}\right)$ (all signals are independent). The values of the object to the agents are

$$
v_{1}(q, c)=q_{1}+q_{2}-c_{1}, \quad v_{2}(q, c)=q_{1}+q_{2}-c_{2} .
$$

There exists no efficient mechanism in this Example. However, there exists a constrainedefficient mechanism, which works as follows. For expositional purposes only, assume that all signals are distributed normally with the mean 0 , and the standard deviation equal to $1 .{ }^{4}$ Denote $t_{i}=q_{i}-c_{i}$. Then

$$
w_{1}\left(t_{1}, t_{2}\right)=t_{1}+\frac{1}{2} t_{2}, \quad w_{2}\left(t_{1}, t_{2}\right)=t_{2}+\frac{1}{2} t_{1}
$$

and sufficient conditions for the existence of a constrained efficient mechanism are satisfied.

Now suppose the auctioneer releases a noisy signal about the common value,

$$
x=q_{1}+q_{2}+\varepsilon
$$

where $\varepsilon$ is distributed normally with the mean 0 and the variance $\sigma^{2}$. Equivalently, the agent $i$ receives a noisy signal $x_{i}=q_{-i}+\varepsilon$ about his opponent's valuation. The constrained-efficient mechanism places the object in the hands of the agent with the highest $\widetilde{w}_{i}\left(t_{1}, t_{2}, x\right)$, where

$$
\widetilde{w}_{i}\left(t_{1}, t_{2}, x\right)=t_{i}+E\left(q_{-i} \mid q_{-i}-c_{-i}=t_{-i}, q_{-i}+\varepsilon=x_{i}\right)=t_{i}+\frac{\sigma^{2}}{1+2 \sigma^{2}} t_{-i}+\frac{\sigma^{2}}{1+2 \sigma^{2}} x_{i} .
$$

The inequality $\widetilde{w}_{1}\left(t_{1}, t_{2}, x\right) \geq \widetilde{w}_{2}\left(t_{1}, t_{2}, x\right)$ is equivalent to

$$
q_{1}-\frac{1+\sigma^{2}}{\sigma^{2}} c_{1} \geq q_{2}-\frac{1+\sigma^{2}}{\sigma^{2}} c_{2}
$$

\footnotetext{
${ }^{4}$ Dasgupta and Maskin (2000) illustration assumes asymmetric uniform distributions.
} 
When the variance $\sigma^{2}$ is finite, this decision rule is more efficient than the one that awards the object to the agent with the highest $q_{i}-c_{i}$. Moreover, the lower is the variance (the higher is the precision of the signal), the higher is efficiency.

Example 2. In this example the environment is the same as described in Example 1. We consider a second-price sealed-bid auction, and assume that the private fixed costs have standard normal distribution, and $q_{1}, q_{2}$ are normally distributed with zero mean and variance $\delta^{2}$. Let the auctioneer announce publicly the variable $q_{1}$. Thus, the second bidder knows for sure how does she value the object, whereas the first receives no additional information. It can be easily seen that a focal point consists of the following two strategies, with the strategy of the second player being weakly dominant and truthful:

$$
b_{1}=q_{1}-\left(1+\delta^{2}\right) c_{1}, \quad b_{2}=q_{1}+q_{2}-c_{2} .
$$

Here more uncertainty (larger $\delta^{2}$ ) leads to larger inefficiency, while revealing information about common component reduces inefficiency.

\section{The Limits}

Unfortunately, second-price sealed-bid auctions are not necessarily constrained-efficient. Theorem 1 could not be extended to cover the case of all second-price sealed bid auctions. ${ }^{5}$ Example 3 below demonstrates how additional information might make an equilibrium in a second-price auction less efficient. ${ }^{6}$

Example 3. There are two agents and one object for sale. The agent's $i$ valuation to the object is $v_{i}=t_{i}+q$, where $t_{i}$ is a private signal observed by $i$, and $q$ is an unknown common component. Assume that $q$ is distributed independently of private signals and normally with the mean $\mu$ and the variance $\delta^{2}$, and assume that the auctioneer knows the private signals of the agents.

Proposition 1 In Example 3, in the absence of additional information, the equilibrium in undominated strategies is efficient. Revelation of information might lead to inefficiency in an equilibrium in undominated strategies.

\footnotetext{
${ }^{5}$ With two bidders, an open assending-bid (English) auction is strategically equivalent to a second-price sealed bid auction. Thus, the same argument is true for English auctions.

${ }^{6}$ The first example with this feature was communicated to us by Alexei Makrushin.
} 
Proof. If no prior information about $q$ is available (the agents know the distribution of $q$ only), then the following strategies constitute a symmetric equilibrium in undominated strategies: $b_{i}=t_{i}+\mu$, which is efficient. Suppose that the auctioneer announces the signal $x=t_{1}+q$. Now there is the following equilibrium in undominated strategies:

$$
\begin{aligned}
& b_{1}=x \\
& b_{2}=t_{2}+E\left(q \mid t_{1}+q=x\right)=\frac{\delta^{2}}{1+\delta^{2}}(x-\mu)+\mu .
\end{aligned}
$$

In the equilibrium, the first agent wins as long as

$$
t_{1}+q>\left(1+\delta^{2}\right) t_{2}+\mu
$$

which might easily be inefficient. (Efficiency requires that the first agent wins when $t_{1}>t_{2}$ ).

In Example 3, agents are asymmetric: the auctioneer's information makes the first agent's private signal public, while bearing no information about the second agent's private signal. ${ }^{7}$ The example might be modified to make agents ex-ante symmetric. For example, the auctioneer might announce either $t_{1}+q$, or $t_{2}+q$ with equal probabilities. ${ }^{8}$ Then in both post-announcement subgames, equilibria are inefficient. So, it is not the ex-ante asymmetry of agents that drives the result in Example 3.

Inefficiency of an auction caused by additional information is not the only problem. Our next example, based on an example from Jackson (1999) shows that it might be that new information might lead to disappearance of a symmetric equilibria at all.

Example 4. There are two bidders in a second-price sealed-bid auction. The agent $i$ values the object at $v_{i}=\frac{1}{2} t_{i}+\frac{1}{2} q$, where $t_{i}$ is a private component, which might be either 0 or 1 with equal probability, and $q$ is a common component, which might be either 0 or $w$ with equal probability. The value of $w$ is unknown to the bidders and might be either $\frac{3}{16}$ with probability $\frac{8}{9}$, or 3 with probability $\frac{1}{9}$.

The agent $i$ learns private signals $t_{i}$ and $s_{i}$, where

$$
\begin{aligned}
& P\left(s_{i}=0 \mid q=0\right)=\frac{3}{4}, \quad P\left(s_{i}=\frac{1}{2} \mid q=0\right)=\frac{1}{4}, \quad P\left(s_{i}=1 \mid q=0\right)=0 \\
& P\left(s_{i}=0 \mid q=w\right)=0, \quad P\left(s_{i}=\frac{1}{2} \mid q=0\right)=\frac{1}{4}, \quad P\left(s_{i}=1 \mid q=w\right)=\frac{3}{4} .
\end{aligned}
$$

Assume that $t_{1}, t_{2}, q$ are independent, and $s_{1}, s_{2}$ are independent conditional on $q$.

\footnotetext{
${ }^{7}$ Assymmetrically informed bidders were studiend in revenue-maximization context in EngelbrechtWiggans et al (1983), see also Krishna (2002, chapter 8).

${ }^{8}$ It is essential that the bidders know whose valuation is announced.
} 
Proposition 2 In Example 4, in the absence of additional information, there exists an efficient symmetric equilibrium. If the auctioneer announces the value of $w$ prior to the auction, no symmetric equilibrium exists.

Proof. If no additional information is available, the following strategies constitute a symmetric equilibrium:

$$
b_{i}\left(t_{i}, s_{i}\right)=\frac{1}{2} t_{i}+\frac{1}{2} s_{i} E w=\frac{1}{2} t_{i}+\frac{1}{4} s_{i} .
$$

The proof of non-existence follows the proof of Proposition 1 in Jackson (1999), and is relegated to the Appendix.

\section{Conclusion}

In this paper, we study the impact of information revelation on efficiency in auctions. If there exists a constrained-efficient mechanism, than any additional information revealed by the auctioneer increases expected efficiency, as measured by the expected value of the object to the winner. We show that this result could not be extended to second-price sealed bid auctions: additional information revealed by the auctioneer might reduce expected equilibrium efficiency, and even lead to non-existence of a symmetric equilibrium. In sum, whereas the inefficiency caused by multidimensionality reduces with revelation of information, inefficiency caused by interdependence between signals agents possess, might increase.

\section{References}

Dasgupta, P. and Maskin, E. (2000) "Efficient Auctions", Quarterly Journal of Economics, 95, 341-388.

Engelbrecht-Wiggans, R., Milgrom, P., and Weber, R. (1983) Competitive Bidding and Proprietary Information, Journal of Mathematical Economics, 11, 161-169.

Goeree, J. and Offerman, T. (1999) "Competitive Bidding in Auctions with Private and Common Values", mimeo.

Jackson M.(1999) "The Non-Existence of Equilibrium in Auctions with Two-Dimensional Types ", mimeo.

Jehiel, P. and Moldovanu, B. (1998), "Efficient Mechanism Design: The Case of Interdependent Valuations", mimeo. 
Krishna, V. (2002) Auction Theory, Academic Press.

Milgrom, P. and Weber, R. (1982), "A Theory of Auctions and Competitive Bidding", Econometrica, 50, 1089-1122.

Pesendorfer, W. and Swinkels, J. (1999) "Efficiency and Information Aggregation in Auctions", CMSEMS discussion paper \# 1168. 


\section{APPENDIX}

Proof of Proposition 2. To complete the proof of Proposition 2, assume that when bidders know $w$, a symmetric equilibrium does exist. For $s_{i}=0$ or $s_{i}=1$, a (weakly) dominant strategy is to bid the expected value as the agent knows whether $q$ equals to 0 or not. That is,

$$
\begin{aligned}
& b(t=0, s=0)=0, \quad b(t=1, s=0)=\frac{1}{2}, \\
& b(t=0, s=1)=\frac{3}{2}, \quad b(t=1, s=1)=2 .
\end{aligned}
$$

Let $t=0, s=1 / 2$. Assume that with non-zero probability, the bid takes value in interval $[0,1 / 2)$. Then the bidder wins, the looser either has $t=0$ and $s=0$ or $t=0$ and $s=1 / 2$. In the first case the expected profit of the winner is zero, but in the second case it is strictly positive, because

$$
E\left(v \mid t=0, s=\frac{1}{2}\right)=\frac{3}{4}>\frac{1}{2} .
$$

So, the agents with $t=0$ and $s=\frac{1}{2}$ have incentives to raise the bid higher than $\frac{1}{2}$. However, if the bidder with $t=0$ and $s=\frac{1}{2}$ submits a bid higher or equal than $\frac{1}{2}$, then the fact that he wins is a bad news, because

$$
E\left(v \mid t=0, s=1 / 2, \text { own bid } \geq \frac{1}{2}\right)=\frac{3}{8}<\frac{1}{2} .
$$

Therefore, there is no symmetric equilibrium. 